\title{
Controllable optical negative refraction and phase conjugation in graphite thin films
}

\author{
Hayk Harutyunyan ${ }^{1,2}$, Ryan Beams ${ }^{1}$ and Lukas Novotny ${ }^{1,3 \star}$
}

\begin{abstract}
Optical metamaterials have demonstrated remarkable physical properties, including cloaking, optical magnetism and negative refraction $^{1-3}$. The last of these has attracted particular interest, mainly because of its promise for super-resolution imaging ${ }^{4-6}$. However, the widespread use of negative refraction at optical frequencies is challenged by high losses and strong dispersion effects, which typically limit operation to narrow frequency bands ${ }^{7}$. Here we use degenerate four-wave mixing to demonstrate controllable negative refraction at a graphite thin film, which acts as a highly efficient phase-conjugating surface. The scheme has very low loss because of the negligible thickness of the nonlinear material and it ensures broadband operation due to the linear band structure of graphene.
\end{abstract}

Negative refraction has been demonstrated with plasmonic materials ${ }^{8,9}$ and with discrete nonlinear elements ${ }^{10}$, but these approaches suffer from high losses and strong dispersion, which restricts operation at optical frequencies to narrow frequency bands ${ }^{7}$. As an alternative approach, it was proposed that a phaseconjugating surface can act as an analogue of a negative-index material boundary and reverse the phase of the electromagnetic fields. By combining a pair of such closely spaced surfaces it is possible to mimic the silver slab originally proposed in ref. 4 and to achieve diffraction-free super-resolution imaging ${ }^{11}$.

Phase conjugation has been used in optics for many decades to recover a beam profile that has been distorted by passing through a perturbative medium ${ }^{12}$. Realization of phase conjugation is based on degenerate four-wave mixing, in which two counterpropagating beams are used to write a holographic grating into a nonlinear crystal, while a third beam is diffracted in a direction exactly opposite to the incident wave. The phase of the diffracted beam is reversed, which is analogous to time-reversed propagation $^{11}$. Typically, phase conjugation is employed to control the field that is reflected from the surface of a nonlinear medium. Here, we use phase conjugation to control the transmitted, that is, negatively refracted field. To achieve negative refraction, wave propagation in the nonlinear medium has to be negligible, which requires a nonlinear medium of vanishing thickness, such as a graphite thin film (Supplementary Information).

The demonstration of phase conjugation in a two-dimensional system is challenging because the nonlinearity is reduced to a single sheet and because all light fields are of the same frequency, which makes the discrimination of linearly scattered background fields difficult. To circumvent these problems, multi-frequency excitation can be used $^{13}$. However, then the refracted beam emerges at a different frequency than the incident signal beam, which destroys any phase relation between input and output. Phase conjugation is not possible with such an approach. In contrast, by using a graphite thin film we are able to achieve negative refraction through phase conjugation, that is, by reversing the phase of the signal beam.

A graphite thin film forms an efficient phase-conjugating surface. Graphene's third-order nonlinear susceptibility has been shown to be two orders of magnitude larger than that of gold ${ }^{14}$ and its atomic smoothness greatly suppresses undesired surface scattering. The high nonlinearity of graphene combined with its relatively low absorption makes it a very attractive optical material for two-dimensional phase conjugation.

As shown in Fig. 1a, a negatively refracting lens is generated by illuminating a graphite thin film with two counter-propagating pump beams $\mathbf{E}_{1} \exp \left(i \mathbf{k}_{1} \cdot \mathbf{r}\right)$ and $\mathbf{E}_{2} \exp \left(i \mathbf{k}_{2} \cdot \mathbf{r}\right)$ of angular frequency $\omega$. A signal beam $\mathbf{E}_{3} \exp \left(i \mathbf{k}_{3} \cdot \mathbf{r}\right)$ of the same frequency is then refracted at the holographic grating written by the two pump beams. This process can be described by a third-order surface nonlinearity $\chi^{(3)}$ that gives rise to nonlinear surface polarization ${ }^{12}$

$$
\begin{aligned}
\mathbf{P}^{(3)}(\omega)= & \varepsilon_{0} \chi^{(3)}(\omega ; \omega, \omega,-\omega) \mathbf{E}_{1} \exp \left(i \mathbf{k}_{1} \mathbf{r}\right) \\
& \times \mathbf{E}_{2} \exp \left(i \mathbf{k}_{2} \mathbf{r}\right) \mathbf{E}_{3}^{*} \exp \left(-i \mathbf{k}_{3} \mathbf{r}\right)
\end{aligned}
$$

For counter-propagating pump beams we have $\mathbf{k}_{1}+\mathbf{k}_{2}=0$ and hence $\mathbf{P}^{(3)}=\mathbf{P}_{0} \exp \left(-i \mathbf{k}_{3} \mathbf{r}\right)=\mathbf{P}_{0} \exp \left(-i k \sin \theta_{3} x\right)$, where $k \sin \theta_{3}$ is the projection of wave vector $\mathbf{k}_{3}$ on the graphite surface. The nonlinear surface polarization $\mathbf{P}^{(3)}$ gives rise to a secondary field

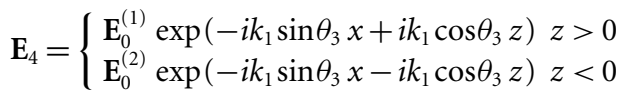

with amplitudes $\mathbf{E}_{0}^{(i)}$ that are determined by $\mathbf{P}_{0}$. As $\mathbf{P}_{0}$ is proportional to the conjugate of $\mathbf{E}_{3}$, the secondary field is the phase conjugate of the signal beam. According to equation (1), the graphite thin film yields not only a phase-conjugate reflected wave that propagates against the incident signal beam, but also a negatively refracted beam, which is a direct consequence of the vanishing thickness of the nonlinear medium.

To verify negative refraction as predicted by equation (1) we have performed the experiment illustrated in Fig. 1. The output of a mode-locked Ti:sapphire laser with centre wavelength $800 \mathrm{~nm}$ and pulse duration of $200 \mathrm{fs}$ is split into three beams, each of which is focused on the sample from a different angle of incidence. The focal spots of the three beams are overlapped on a graphite thin film sandwiched between two glass hemispheres. A low-numericalaperture multi-mode fibre picks up the refracted light and sends it to a photodetector. To increase the signal-to-noise ratio and reject spuriously scattered light, the intensity of one of the beams is modulated with an optical chopper and the detected signal is

${ }^{1}$ Institute of Optics, University of Rochester, Rochester, New York 14627, USA, ${ }^{2}$ Center for Nanoscale Materials, Argonne National Laboratory, Argonne, Illinois 60439, USA, ${ }^{3}$ ETH Zürich, Photonics Laboratory, 8093 Zürich, Switzerland. *e-mail: Inovotny@ethz.ch 


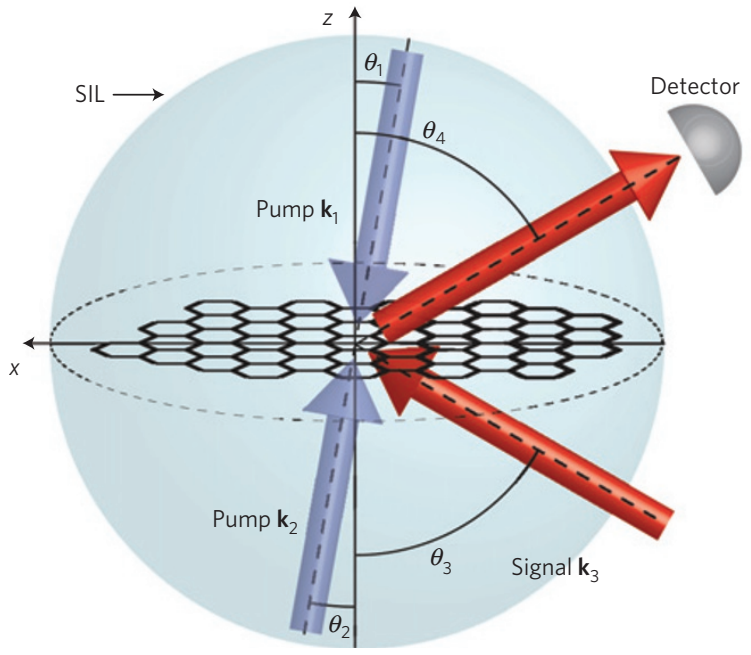

Figure 1 | Illustration of the experiment. Two pump beams (blue arrows) are focused on a graphite thin film (honeycomb lattice) at angles $\theta_{1}=\theta_{2}$ writing a holographic grating. The signal beam (lower red arrow) is refracted in the negative direction (upper red arrow) and is detected by a photodetector. The graphite thin film is sandwiched between two hemispherical SILs to ensure that sample rotation does not affect the beam alignment.

demodulated with a lock-in amplifier. The temporal overlap of the laser pulses has been optimized by adjusting the optical path length of the beams and observing the blueshifted multi-photon excited emission from the graphite thin film ${ }^{15,16}$.

Samples of graphite thin films were prepared on a fusedsilica glass slide by micromechanical cleavage ${ }^{17}$. Suitable flakes were identified with an optical microscope and subsequently characterized by confocal transmission microscopy. The thickness and quality of the graphite films were determined by atomic force microscopy. Graphite flakes consisting of $\sim 20$ atomic graphene layers were selected because they provide the best compromise between nonlinear signal generation and absorption ${ }^{14,18}$. However, we have verified that negative refraction can also be measured on graphite thin films with fewer layers (Supplementary Information). The selected flakes have a lateral size that is significantly larger than the laser spot sizes of $\sim 10 \mu \mathrm{m}$. A drop of immersion oil is deposited on top of the flake and a second fused-silica glass slide is placed over it. Finally, sub-hemispherical solid immersion lenses (SIL) are bonded to each of the two glass slides and centred over the graphite thin film to ensure that sample rotation does not affect the alignment of the excitation beams (Fig. 1). The sample is then fixed on a rotation stage that is used for tuning the angles of incidence $\theta_{i}$ of the excitation beams. The rotation stage is supported by a translation stage for positioning the flake into the focal region of the excitation beams.

Figure 2 shows our experimental results. The refracted intensity exhibits a resonance at $\theta_{3}-\theta_{4} \approx 0^{\circ}$; that is, the signal beam is refracted in the negative direction as predicted by equation (1). The angular width of the measured curve is defined by the numerical aperture of the external focusing lenses. The signal disappears if one of the excitation beams is delayed in time (curve at the bottom in Fig. 2a). The remaining linear background signal originates from reflections from the outer SIL surfaces.

The dependence of the negatively refracted intensity on the time delay of the signal beam is explicitly shown in Fig. 2b. Here, the sample rotation angle is fixed at $\theta_{3}-\theta_{4}=0^{\circ}$ and the signal beam is delayed by adjusting the path length using a translation stage. The resulting signal pulses exhibit a mean pulse duration of $\sim 3 \mathrm{ps}$, which is more than an order of magnitude longer than the duration of the

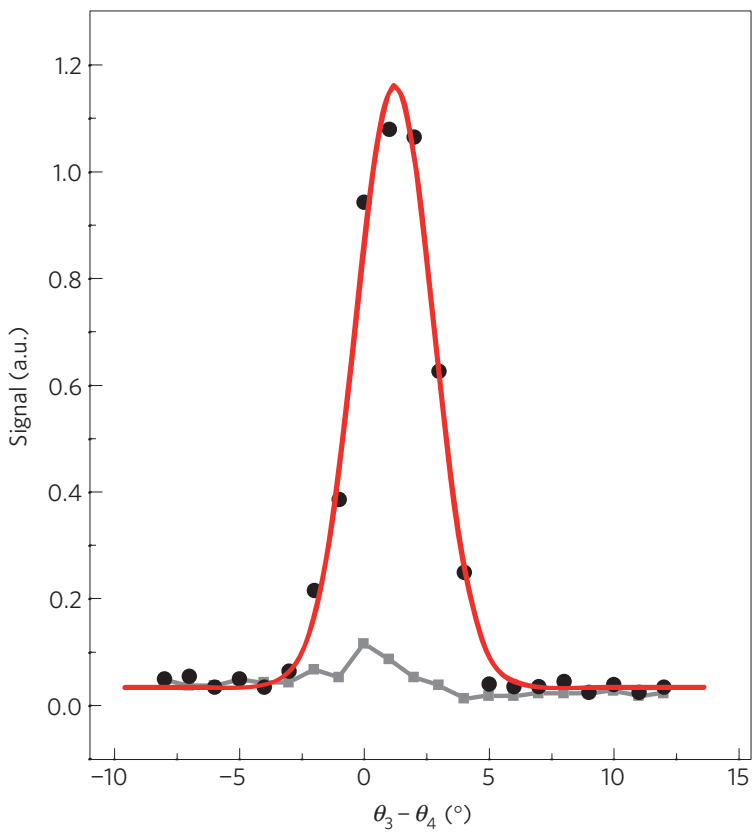

b

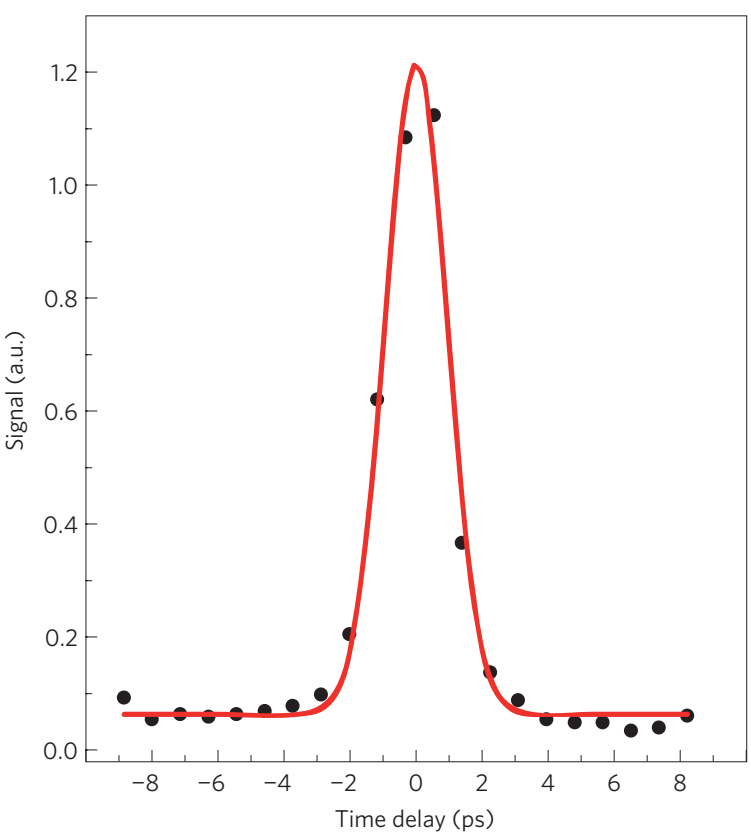

Figure 2 | Experimental verification. $\mathbf{a}$, The intensity of the refracted light exhibits a peak at $\theta_{4} \approx \theta_{3}$, in agreement with negative refraction. The grey curve is a control measurement obtained by time-delaying the laser pulses of one of the pump beams. $\mathbf{b}$, Negatively refracted signal intensity at $\theta_{3}=\theta_{4}$ as a function of time delay of the signal beam laser pulses.

initial laser pulses. The increased width is the result of dispersion; that is, the pulses get stretched in time as they travel through the experiment. This has been indeed confirmed by measuring the autocorrelation function of the pulses using the multi-photon excited luminescence as a signal (data not shown). For $10 \mathrm{~mW}$ of laser power in each of the excitation beams, the efficiency of negative refraction is found to be $0.1 \%$, which is remarkably high for a nonlinear process. The efficiency can be improved with higher laser powers and tighter focusing, approaching unity for realistic intensity levels. For example, focusing the incident beams to spots of $\sim 600 \mathrm{~nm}$ or compressing the pulse durations at the sample to $100 \mathrm{fs}$ will provide sufficiently high intensities for unity conversion, 


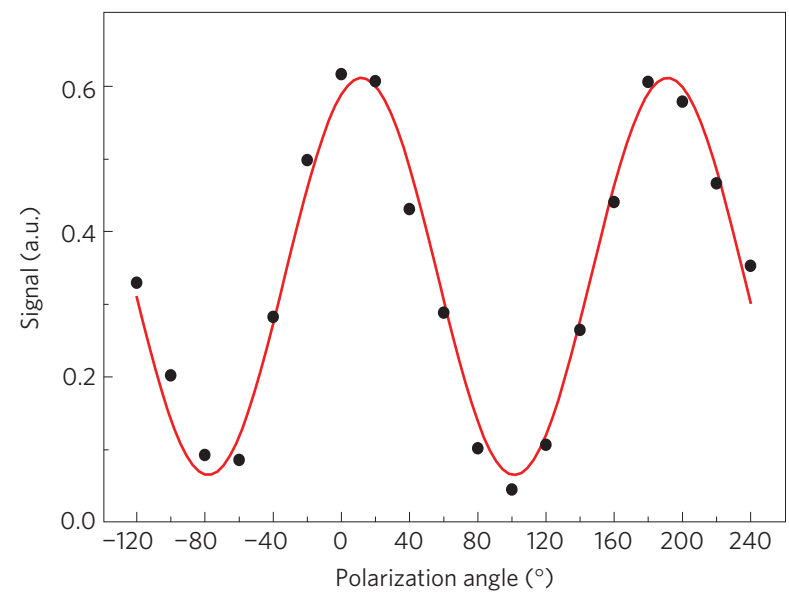

Figure 3 | Polarization dependence of negatively refracted signal. The polarization of one pump beam is rotated while the polarizations of the other pump beam and the signal beam are fixed at $0^{\circ}$ (s-polarization). A polarizer in the detection path selects the s-polarized component of the negatively refracted signal.

while keeping the power densities well below the damage threshold of graphite thin films ${ }^{19}$. Note that the efficiency of signal conversion does not depend on how the intensity is distributed among the incident laser beams. Therefore, by significantly increasing the power of the pump beams we can reach unity conversion with much lower signal beam intensities.

The polarization of the negatively refracted signal is defined by the polarizations of signal and pump beams and the symmetry of the $\chi^{(3)}$ nonlinear susceptibility tensor of graphite thin films. We have measured this polarization dependence by sending the signal and pump beams through waveplates to rotate the incident polarizations and placing a polarizer in front of the detector to analyse the polarization of the refracted beam. The data in Fig. 3 show the measurement outcome of rotating the polarization of one of the pump beams while keeping the other beams in the $s$-polarized state. Evidently, the signal is at a maximum when the pump beam is $s$-polarized as well, implying that the thirdorder nonlinearity of graphite thin films is associated with inplane fields ${ }^{18}$. Similar measurements can be performed with other polarizer settings to trace out the components of the $\chi^{(3)}$ tensor of graphite thin films.

As the nonlinear response of graphite thin films is driven by in-plane fields, the tensor components $\chi_{i j k l}^{(3)}$ are non-zero only if $i, j, k, l \in\{x, y\}$. Furthermore, taking into account the $D_{6 v}$ symmetry of graphene the number of non-zero tensor elements is reduced to 8 , of which only 3 are independent ${ }^{12}$. The maxima of the curve in Fig. 3 are proportional to $\chi_{x x x x}^{(3)}$, whereas the minima indicate that $\chi_{x x y x}^{(3)}$ is zero (after subtraction of background). With other polarizer settings we can trace out other tensor components. The highest values are found for $\chi_{x x x x}^{(3)}$ and $\chi_{y y y y}^{(3)}$. Mixed terms such as $\chi_{y x y x}^{(3)}$ or $\chi_{y y x x}^{(3)}$ are smaller by roughly a factor of $1 / 3$, and terms with only one $x$ or one $y$ index, for example, $\chi_{y x x x}^{(3)}$ or $\chi_{y y x y}^{(3)}$, are zero. We have also measured the dependence of the signal intensity on excitation power and confirmed that the negatively refracted signal depends linearly on the power of each excitation beam (data not shown).

Our experimental results are the first demonstration of optical negative refraction based on phase conjugation and time-reversal.
The scheme provides all-optical control of negative refraction, which can be exploited for many optoelectronic applications. Using graphite thin films as an interface material ensures low-loss operation and virtually unlimited bandwidth.

Received 24 October 2012; accepted 3 April 2013; published online 5 May 2013; corrected online 10 May 2013

\section{References}

1. Schurig, D. et al. Metamaterial electromagnetic cloak at microwave frequencies. Science 314, 977-980 (2006).

2. Smith, D. R., Pendry, J. B. \& Wiltshire, M. C. K. Metamaterials and negative refractive index. Science 305, 788-792 (2004).

3. Soukoulis, C. M. \& Wegener, M. Past achievements and future challenges in the development of three-dimensional photonic metamaterials. Nature Photon. 5, 523-530 (2011).

4. Pendry, J. B. Negative refraction makes a perfect lens. Phys. Rev. Lett. 85, 3966-3969 (2000).

5. Fang, N., Lee, H., Sun, C. \& Zhang, X. Sub-diffraction-limited optical imaging with a silver superlens. Science 308, 534-537 (2005).

6. Taubner, T., Korobkin, D., Urzhumov, Y., Shvets, G. \& Hillenbrand, R. Near-field microscopy through a sic superlens. Science 313, 1595 (2006).

7. Stockman, M. Criterion for negative refraction with low optical losses from a fundamental principle of causality. Phys. Rev. Lett. 98, 177404 (2007).

8. Lezec, H. J., Dionne, J. A. \& Atwater, H. A. Negative refraction at visible frequencies. Science 316, 430-432 (2007).

9. Valentine, J. et al. Three dimensional optical metamaterial exhibiting negative refractive index. Nature 455, 376-379 (2008).

10. Katko, A. R. et al. Phase conjugation and negative refraction using nonlinear active metamaterials. Phys. Rev. Lett. 105, 123905 (2010).

11. Pendry, J. B. Time reversal and negative refraction. Science 322, 71-73 (2008).

12. Boyd, R. W. Nonlinear Optics 3rd edn (Academic, 2008).

13. Palomba, S. et al. Optical negative refraction by four-wave mixing in thin metallic nanostrucutres. Nature Mater. 11, 34-38 (2012).

14. Hendry, E., Hale, P., Moger, J., Savchenko, A. \& Mikhailov, S. Coherent nonlinear optical response of graphene. Phys. Rev. Lett. 105, 097401 (2010).

15. Lui, C. H., Mak, K. F., Shan, J. \& Heinz, T. F. Ultrafast photoluminescence from graphene. Phys. Rev. Lett. 105, 127404 (2010).

16. Liu, W-T. et al. Nonlinear broadband photoluminescence of graphene induced by femtosecond laser irradiation. Phys. Rev. B 82, 081408(R) (2010).

17. Novoselov, K. S. et al. Electric field effect in atomically thin carbon films. Science 306, 666-669 (2004).

18. Mikhailov, S. A. Theory of the nonlinear optical frequency mixing effect in graphene. Physica E 44, 924-927 (2012).

19. Roberts, A. et al. Response of graphene to femtosecond high-intensity laser irradiation. Appl. Phys. Lett. 99, 051912 (2011).

\section{Acknowledgements}

This research was financially supported by the US Department of Energy (grant DE-FG02-05ER46207). We thank J. Pendry, B. Deutsch, P. Bharadwaj and A. Jorio for valuable input and fruitful discussions. H.H. acknowledges financial support during the completion of the manuscript from the Center for Nanoscale Materials, which is supported by the US Department of Energy, Office of Science, Office of Basic Energy Sciences, under Contract No. DE-AC02-06CH11357.

\section{Author contributions}

H.H. built the experimental set-up, performed the measurements, analysed the data and contributed to sample fabrication, R.B. fabricated and characterized the samples, L.N. conceived and supervised the project and analysed the data. All authors contributed to discussions and the writing of the manuscript.

\section{Additional information}

Supplementary information is available in the online version of the paper. Reprints and permissions information is available online at www.nature.com/reprints. Correspondence and requests for materials should be addressed to L.N.

\section{Competing financial interests}

The authors declare no competing financial interests. 


\section{CORRIGENDUM}

\section{Controllable optical negative refraction and phase conjugation in graphite thin films}

Hayk Harutyunyan, Ryan Beams and Lukas Novotny

Nature Physics http://dx.doi.org/10.1038/nphys2618 (2013); published online 5 May 2013; corrected online 10 May 2013.

In the version of this Letter originally published online, the $y$ axis in Fig. $2 \mathrm{~b}$ was aligned incorrectly. This error has now been corrected in all versions of the Letter. 